\title{
A new synthesis procedure for linear-phase paraunitary digital filter banks
}

\author{
L. Chen, K. P. Chan \\ Department of Computer Science \\ University of Hong Kong
}

\author{
T. Q. Nguyen \\ Department of Electrical Computer Engineering \\ University of Wisconsin, Madison
}

\author{
Y. Zheng \\ Institute of AI \& PR \\ University of Shantou, China
}

\begin{abstract}
In this paper, a new design algorithm is presented for a family of linear phase paraunitary filter banks with generalized filter length and symmetric polarity. A number of new constraints on the distributions of filter length and symmetry polarity among the channels are derived. In the algorithm, the lengths of the filters are gradually reduced through a cascade of lattice structures. The derivations for filter banks with even and odd number of channels are formulated in a unified form.
\end{abstract}

\section{Introduction}

Recently, there has been considerable interest among researchers in the design of $M$-channel maximally decimated filter banks, as shown in Fig. 1. The

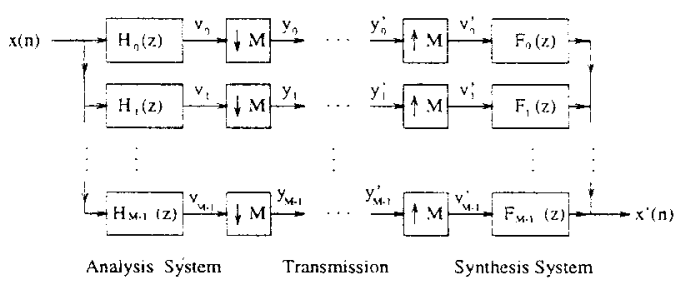

Figure 1: $M$-Channel Analysis/Synthesis Systems

theory of linear-phase (LP) filter banks and their applications have been addressed by many authors. In two-channel case, linear phase (symmetry) property has been imposed on the traditional subband filter banks [1, 2, 3]. It was shown that among all cases of two-channel FIR perfect-reconstruction QMF structures which yield LP filters, only two cases yield good filters in the practical sense $[4,5]$. The results are extended to the case of arbitrary number $M$ of channels in $[6,7]$. In parallel to the work on the general perfect-reconstruction filter banks, which are biorthogonal, researches have also been done for paraunitary systems. Lattice factorizations were developed in $[8,10]$ for even $M$. In $[11,12,13]$, the results were extended to the case where $M$ is odd.

In the lattice factorizations of $[8,11]$, the filter length is restricted to $N=k M$ or $2 k M$ for even and odd $M$, and the step size in increasing the filter length is at least $M$. This is not convenient when the number of channels is large, e.g., $M=8$ or 16 . In the phase of filter design, an increase of filter length by a large $M$ implies a much higher non-linear parameter space to be searched and the optimization program tends to be trapped in local minima. In the phase of implementing filter banks, i.e., in the analysis/synthesis systems, a large increase in filter length by $M$ gives much higher computational complexity. This leads to the design of general equal length filter banks and unequal length filter banks.

In [14], the theory and structures are studied for a large subclass of $M$-channel LP perfectreconstruction FIR filter banks whose the analysis and synthesis filters have length $L_{i}=k_{i} M+\beta$, where $\beta$ is an arbitrary integer, $0 \leq \beta<M$, and $k_{i}$ is a non-negative integer. The extension of filter length from $N=k M$ to $k_{i} M+\beta$ gives more flexibility in fine-tuning filter length to meet a given filter specification, e.g., stopband attenuation. The unified $\beta$ leads to this class of filter banks to have a simple characterization of symmetric property in polyphase representation (Eq. (2.1) in [8] and Eq. (3) in [14]). It is noted that tree-structured filter banks $[2,3]$ can be viewed as a kind of unequal length filter banks. In terms of lapped orthogonal transforms [9, 15, 16], the freedom in filter length provides the possibility of overlap which is a fraction of the number of channels $M$. Moreover, it has been shown that this class of filter banks can be used in processing finite-length signal with the symmetric extension method $[17,18]$.

In this paper, we further investigate factorizations of linear-phase paraunitary systems. In Section II, several known results are reviewed and new constraints are derived. Section III and IV show that the filter banks can be designed by a successive of length reductions through a cascade of lattice structures which is minimal in terms of the number of delays used for implementation. The algorithm can also be applied to filter banks where the filter length is an integral multiple of the number of channels, or, filter banks with equal length. In Section V, a brief summary is given and a design example is included to verify the theory. 


\section{Basic constraints and properties}

Definition 2.1 Consider an $M$-channel linearphase filter bank where among the $M$ filters, there are $\mu_{k}+\nu_{k}$ filters that have length $k M+\beta, 0 \leq k<$ $K$, where $\mu_{k}$ and $\nu_{k}$ stand that the numbers of symmetric and anti-symmetric filters, respectively. A matrix $\kappa$-stack is defined to indicate distributions of the length and symmetry polarity among the filters

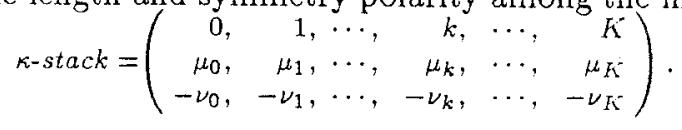

P'roposition 2.1 In an $M$-channel LP perfectreconstruction filter bank with filter lengths $L_{i}=$ $k_{i} M+\beta, 0 \leq k_{i} \leq K$, let $\psi=\sum_{i=0}^{M-1} k_{i}, \phi=$ $\sum_{k=0}^{K} \mu_{k}, \varphi=\sum_{k=0}^{K} \nu_{k}$. The following results have been shown $[8,14]$

1. If $M \in Z_{e}$ and $\beta \in Z_{e}, \quad \psi \in Z_{e}, \phi=\frac{M}{2}, \varphi=\frac{M}{2}$;

2. If $M \in Z_{e}$ and $\beta \in Z_{o}, \quad \psi \in Z_{o}, \phi=\frac{M}{2}+1, \varphi=\frac{M}{2}-1$;

3. If $M \in Z_{o}$ and $\beta \in Z_{e}, \quad \psi \in Z_{o}, \phi=\frac{M+1}{2}, \varphi=\frac{\frac{M}{2}-1}{2}$;

4. If $M \in Z_{o}$ and $\beta \in Z_{o}, \quad \psi \in Z_{e}, \phi=\frac{M^{2}+1}{2}, \varphi=\frac{M^{2}-1}{2}$;

where $Z_{\varepsilon}$ and $Z_{o}$ denote the sets of even and odd integers, respectively.

Proposition 2.1 shows the total sum of the filter length and the total numbers of symmetric and antisymmetric filters. However, the permissible length and symmetry polarity distributions are not given. For example, for a given total number $\mu_{K}+\nu_{K}$ of filters that have the longest length $K M+\beta$, the relation for the number of symmetric filters $\mu_{K}$ and the number of anti-symmetric filters $\nu_{K}$ has not been shown. In the following, new results will be presented and lead to the unification of the lattice structures of filter banks with even and odd number of channels.

Partitioning of $\mathbf{E}_{K}(z)$ : It is noted that the perfect-reconstruction property is preserved in spite of the interchange of rows of the polyphase matrix $\mathbf{E}_{K}(z)$. With row-wise permutations, $\mathbf{E}_{K}(z)$ can always be partitioned as

$$
\mathbf{E}_{K}(z)=\left(\begin{array}{c}
\mathbf{E}_{s}(z) \\
\mathbf{E}_{a}(z) \\
\mathbf{E}_{d}(z)
\end{array}\right)
$$

where $\mathbf{E}_{s}(z)$ and $\mathbf{E}_{a}(z)$ represent $\mu_{K}$ symmetric and $\nu_{K}$ anti-symmetric filters with the longest length $L_{i}=K M+\beta ; \mathbf{E}_{d}(z)$ stands for the rest $M-$ $\left(\mu_{K}+\nu_{K}\right)$ filters. For convenience, the subsequent discussions assume that $\mathbf{E}_{K}(z)$ has already been arranged in the form of (2). Let $\mathbf{E}_{K}(z)$ be expressed as $\mathbf{E}_{K}(z)=\mathrm{e}(0)+\cdots+\mathrm{e}(K-1) z^{-(K-1)}+$ $\mathrm{e}(K) z^{-K}, \mathrm{e}(K) \neq 0$, and $\mathrm{e}(0), \mathrm{e}(K-1)$ and $\mathrm{e}(K)$ are partitioned as

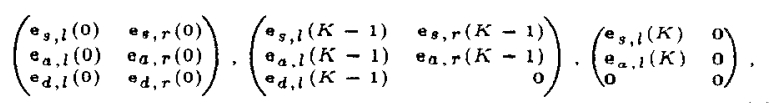

where the left hand side part consists of $\beta$ columns; and the right hand side part consists of $M-\beta$ columns. The lower part of $\mathrm{e}(K)$ and $\mathrm{e}_{d, r}(K-1)$, which is to the right of $\mathbf{e}_{d, l}(K-1)$, are set to 0 , as the corresponding filters are shorter than $K M+\beta$. Also, since the maximum length of filters are $K M+\beta$, all the elements on the right hand side of $\mathrm{e}(K)$ are set to zeros.

Proposition 2.2 For a LP paraunitary filter bank $\mathbf{E}_{K}(z)$, the polyphase components $\mathbf{e}_{s}(0)$ and $\mathbf{e}_{a}(0)$ as defined in (3) satisfy

$$
\begin{aligned}
& \operatorname{rank}\left(\mathrm{e}_{3}(0)\right)=\operatorname{rank}\left(\mathrm{e}_{a}(0)\right), \quad 2 \leq K \\
& \operatorname{rank}\left(\mathrm{e}_{s, l}(0)\right)=\operatorname{rank}\left(\mathrm{e}_{a, l}(0)\right), \quad 1 \leq K .
\end{aligned}
$$

Corollary 2.1 For an $M$-channel LP paraunitary filter bank with filter lengths $L_{i}=k_{i} M+\beta, 0 \leq \beta \leq$ $M-1,0 \leq k_{i} \leq K$. The filters with the longest length $L_{i}=K M+\beta$ can not be all symmetric or all anti-symmetric.

Proposition 2.3 Let $\mathbf{E}_{1}(z)$ be a FIR LP paraunitary filter bank whose $\kappa$-stack is $\left(\begin{array}{cc}0, & 1 \\ \mu_{0}, & \mu_{1} \\ -\nu_{0}, & -\nu_{1}\end{array}\right)$ Then,

$\mu_{0}-\nu_{0}=\left\{\begin{array}{l}0, \text { if } \beta \text { is even, } \\ 1, \text { if } \beta \text { is odd, }\end{array} \quad \mu_{1}-\nu_{1}=\left\{\begin{array}{l}0, \text { if } M-\beta \text { is even, } \\ 1, \text { if } M-\beta \text { is odd. }\end{array}\right.\right.$

i.e., the numbers of symmetric and anti-symmetric filters are balanced.

\section{Lattice factorization}

Based on Proposition 2.2, we show the "order reduction" process to $K=1$. The next section will present a general initialization of the lattice structure.

Proposition 3.1 Let $\mathrm{E}_{K}(z), 2 \leq K$ be a FIR LP paraunitary filter bank whose $\kappa$-stack is

$\left(\begin{array}{rrrrrrr}0, & 1, & 2, & \cdots, & K-2, & K-1, & K \\ \mu_{0}, & \mu_{1}, & \mu_{2}, & \cdots, & \mu_{K-2}, & \mu_{K-1}, & \mu_{K} \\ -\nu_{0}, & -\nu_{1}, & -\nu_{2}, & \cdots, & -\nu_{K-2}, & -\nu_{K-1}, & -\nu_{K}\end{array}\right)$.

Then it can always be factored into a left hand side factor $\boldsymbol{\Xi}_{K}(z)$ and a LP paraunitary filter bank $\mathbf{E}_{K-1}(z)$ with the order $K$ reduced by $1, \mathbf{E}_{K}(z)=$ $\mathbf{\Xi}_{K}(z) \mathbf{E}_{K-1}(z)$, where the $\kappa$-stack of $\mathbf{E}_{K-1}(z)$

$$
\left(\begin{array}{rrrrrr}
0, & 1, & 2, & \cdots, & K-2, & K-1 \\
\mu_{0}^{\prime}, & \mu_{1}^{\prime}, & \mu_{2}^{\prime}, & \cdots, & \mu_{K-2}^{\prime}, & \mu_{K-1}^{\prime} \\
-\nu_{0}^{\prime}, & -\nu_{1}^{\prime}, & -\nu_{2}^{\prime}, & \cdots, & -\nu_{K-2}^{\prime}, & -\nu_{K-1}^{\prime}
\end{array}\right),
$$

is related to that of $\mathbf{E}_{K}(z)$ in (7) as

$$
\left\{\begin{array}{lll}
\mu_{k}^{\prime}=\mu_{k}+\varsigma, & & k=K-1 \\
\nu_{k}^{\prime}=\nu_{k}+\varsigma, & & k=K-1 \\
\mu_{k}^{\prime}=\mu_{k}+\mu_{k+1}-\varsigma, & k=K-2 \\
\nu_{k}^{\prime}=\nu_{k}+\nu_{k+1}-\varsigma, & k=K-2 \\
\mu_{k}^{\prime}=\mu_{k}, & & 0 \leq k \leq K-3 \\
\nu_{k}^{\prime}=\nu_{k}, & & 0 \leq k \leq K-3
\end{array}\right.
$$

where $\varsigma=\operatorname{rank}\left(\mathbf{e}_{s}(0)\right)$.

Proof: The proof is achieved through an order reduction process and is divided into three steps.

i) Partitioning of $\mathbb{E}_{s}(z)$ and $\mathbf{E}_{a}(z)$ : Let $\mathbf{E}_{s}(z)$ be expressed as $\mathbf{E}_{s}(z)=\mathbf{e}_{s}(0)+\mathbf{e}_{s}(1) z^{-1}+\cdots+$ $\mathrm{e}_{s}(K) z^{-K}, \mathrm{e}_{s}(K) \neq 0$. Denote the rank of $\mathrm{e}_{s}(0)$ as 
ऽ. By cascading an orthonormal matrix $\mathbf{U}_{\mu_{K}}, \mathbf{E}_{3}(z)$ can be decomposed as $\left(\begin{array}{c}\mathbf{E}_{s}^{\prime}(z) \\ \mathbf{E}_{s}^{\prime \prime}(z)\end{array}\right)=\mathbf{U}_{\mu_{K}} \mathbf{E}_{s}(z)$, where

$$
\begin{aligned}
& \mathbf{E}_{s}^{\prime}(z)=\mathrm{e}_{s}^{\prime}(0)+\mathrm{e}_{s}^{\prime}(1) z^{-1}+\cdots+\mathrm{e}_{s}^{\prime}(K-1) z^{-(K-1)}+\mathrm{e}_{s}^{\prime}(K) z^{-K} \\
& \mathbf{E}_{s}^{\prime \prime}(z)=0+\mathbf{e}_{s}^{\prime \prime}(1) z^{-1}+\cdots+\mathrm{e}_{s}^{\prime \prime}(K-1) z^{-(K-1)}+0
\end{aligned}
$$

$\mathbf{E}_{s}^{\prime}(z)$ consists of $\varsigma$ symmetric filters and all the rows of the polyphase components $\mathbf{e}_{s}^{\prime}(0)$ are linearly independent, i.e., $\operatorname{rank}\left(\mathbf{e}_{s}^{\prime}(0)\right)=\varsigma ; \mathbf{E}_{s}^{\prime \prime}(z)$ represents the rest $\mu_{K}-\varsigma$ symmetric filters with $\mathbf{e}_{s}^{\prime \prime}(0)$ set to 0 through linear combinations. Likewise, we can express $\mathbf{E}_{a}(z)$ as $\left(\begin{array}{c}\mathbf{E}_{a}^{\prime}(z) \\ \mathbf{E}_{a}^{\prime \prime}(z)\end{array}\right)=\mathbf{V}_{\nu_{K}} \mathbf{E}_{a}(z)$. Hence,

$$
\mathbf{E}_{K}(z)=\mathbf{Q}_{1}\left(\begin{array}{c}
\mathbf{E}_{s}^{\prime}(z) \\
\mathbf{E}_{s}^{\prime \prime}(z) \\
\mathbf{E}_{a}^{\prime}(z) \\
\mathbf{E}_{a}^{\prime \prime}(z) \\
\mathbf{E}_{d}(z)
\end{array}\right)=\mathbf{Q}_{1} \mathbf{Q}_{2}\left(\begin{array}{c}
\mathbf{E}_{g}^{\prime}(z) \\
\mathbf{E}_{a}^{\prime}(z) \\
\mathbf{E}_{c}^{\prime \prime}(z) \\
\mathbf{E}_{a}^{\prime \prime}(z) \\
\mathbf{E}_{d}(z)
\end{array}\right),
$$

where $\mathbf{Q}_{1}=\operatorname{diag}\left(\mathbf{U}_{\mu_{K}}^{T}, \mathbf{V}_{\nu_{K}}^{T}, \mathbf{I}_{M-\mu_{K}-\nu_{K}}\right)$. and $\mathbf{Q}_{2}$ exchanges $\mathbf{E}_{s}^{\prime \prime}(z)$ and $\mathbf{E}_{a}^{\prime}(z)$.

ii) Reduction of the order of $\mathbf{E}_{s}^{\prime}(z)$ and $\mathbf{E}_{a}^{\prime}(z)$ by $M$ : Proposition 2.2 shows that $\operatorname{rank}\left(\mathbf{e}_{a}(0)\right)=$ $\operatorname{rank}\left(\mathbf{e}_{s}(0)\right)=\varsigma$. Pairwisely combining the $\varsigma$ symmetric and $\varsigma$ anti-symmetric rows in $\mathrm{E}_{s}^{\prime}(z)$ and $\mathbf{E}_{a}^{\prime}(z)$ yields $\mathbf{F}_{K}(z)$ satisfying the pairwise time-reversed property

$$
\mathbf{F}_{K}(z)=\mathbf{T}(\varsigma)\left(\begin{array}{l}
\mathbf{E}_{s}^{\prime}(z) \\
\mathbf{E}_{a}^{\prime}(z)
\end{array}\right), \quad \mathbf{T}(\varsigma)=\frac{1}{\sqrt{2}}\left(\begin{array}{cc}
\mathbf{I}_{\varsigma} & \mathbf{J}_{\varsigma} \\
\mathbf{J}_{\varsigma} & -\mathbf{I}_{\varsigma}
\end{array}\right) .
$$

It can be shown that $\mathbf{F}_{K}(z)$ can be factored into the "reduced form" $\mathrm{F}_{K-1}(z)$

$$
\mathbf{F}_{K}(z)=\mathbf{A}_{K}(z) \mathbf{F}_{K-1}(z), \quad \mathbf{A}_{K}(z)=\Lambda(z) \mathbf{W}_{K}
$$

where $\mathbf{W}_{K}=\frac{1}{2}\left(\begin{array}{cc}\mathbf{I}_{\varsigma} & \mathbf{I}_{\varsigma} \\ \mathbf{J}_{\varsigma} & -\mathbf{J}_{\varsigma}\end{array}\right)\left(\begin{array}{ll}\mathbf{U}_{\varsigma} & \\ & \mathbf{V}_{\varsigma}\end{array}\right)\left(\begin{array}{cc}\mathbf{I}_{\varsigma} & \mathbf{J}_{\varsigma} \\ \mathbf{I}_{\varsigma}-J_{\varsigma}\end{array}\right) ; \boldsymbol{\Lambda}(z)=$ $\left(\mathbf{I}_{\varsigma}{ }_{z^{-1} \mathbf{I}_{\varsigma}}\right) ; \quad \mathbf{U}_{\varsigma}$ and $\mathbf{V}_{\varsigma}$ are two orthonormal matrices. Moreover, $\mathbf{F}_{K-1}(z)$ with pairwise timereversed property can be expressed as $\mathbf{F}_{K-1}(z)=$ $\mathbf{T}(\varsigma)\left(\begin{array}{c}\mathbf{E}_{s}^{\prime \prime \prime}(z) \\ \mathbf{E}_{a}^{\prime \prime \prime}(z)\end{array}\right)$, where $\mathbf{E}_{s}^{\prime \prime \prime}(z)$ and $\mathbf{E}_{a}^{\prime \prime \prime}(z)$ are LP filters with symmetric and anti-symmetric property, respectively. Hence,

$$
\left(\begin{array}{c}
\mathbf{E}_{s}^{\prime}(z) \\
\mathbf{E}_{a}^{\prime}(z)
\end{array}\right)=\mathbf{A}^{\prime}(z)\left(\begin{array}{c}
\mathbf{E}_{s}^{\prime \prime \prime}(z) \\
\mathbf{E}_{a}^{\prime \prime \prime}(z)
\end{array}\right)
$$

where $\mathbf{A}^{\prime}(z)=\mathbf{T}(\varsigma) \mathbf{A}(z) \mathbf{T}(\varsigma)$. It yields the first and second equations in (9).

iii) Reduction of the order of $\mathrm{E}_{s}^{\prime \prime}(z)$ and $\mathrm{E}_{a}^{\prime \prime}(z)$ by $2 M:$ As shown in (11), the first $M$ taps of the filters in $\mathrm{E}_{s}^{\prime \prime}(z)$ are set to zero. Due to the symmetry of $\mathrm{E}_{s}^{\prime \prime}(z)$, the last (highest order) $M$ taps are also zeros. As a result, $\mathbf{E}_{s}^{\prime \prime}(z)$ can be shifted to the left by $z$, resulting in a reduction in length of $2 M, \mathbf{E}_{d}^{\prime \prime}(z)=$ $z^{-1} \mathbf{E}_{d}^{\prime \prime \prime \prime}(z)$. Similarly, $\mathbf{E}_{a}^{\prime \prime}(z)$ can be shifted to the left by $z$ to lower order filters $\mathbf{E}_{a}^{\prime \prime \prime \prime}(z)$, namely, $\mathbf{E}_{a}^{\prime \prime}(z)=$ $z^{-1} \mathbf{E}_{a}^{\prime \prime \prime \prime}(z)$. It gives the third and fourth equations in $(9)$.
Substituting the results in Step (ii) and (iii) gives the factorization $\mathrm{E}_{K}(z)=\mathrm{Q}_{1} \mathrm{Q}_{2} \boldsymbol{\Lambda}^{\prime}(z) \mathrm{E}_{K-1}(z)$ where $\boldsymbol{\Lambda}^{\prime}(z)=$ $\operatorname{diag}\left(\mathbf{A}^{\prime}(z), z^{-1} \mathbf{I}_{\mu_{K}-\varsigma}, z^{-1} \mathbf{I}_{\nu_{K}-\varsigma}, \mathbf{I}_{M-\mu_{K}-\nu_{K}}\right)$ and

$$
\mathbf{E}_{K-1}(z)=\left(\begin{array}{l}
\mathbf{E}_{s}^{\prime \prime \prime}(z) \\
\mathbf{E}_{a}^{\prime \prime \prime}(z) \\
\mathbf{E}_{s}^{\prime \prime \prime \prime}(z) \\
\mathbf{E}_{a}^{\prime \prime \prime \prime}(z) \\
\mathbf{E}_{d}(z)
\end{array}\right)
$$

\section{Initialization}

Proposition 4.1 Let $\mathrm{E}_{1}(z)$ be a FIR LP paraunitary matrix whose $\kappa$-stack is $\left(\begin{array}{rr}0, & 1 \\ \mu_{0}, & \mu_{1} \\ -\nu_{0}, & -\nu_{1}\end{array}\right)$. Then it can always be factored into a right hand side factor $\Theta(z)$ and an initial matrix $\Omega_{0}$

$$
\mathrm{E}_{1}(z)=\Omega_{0} \Theta(z)
$$

where $\Omega_{0}=\left(\begin{array}{ccc}\mathbf{U} & & \\ & \mathbf{V} & \\ & & \mathbf{W}\end{array}\right) . \mathbf{U}, \mathbf{V}$ and $\mathbf{W}$ are orthonormal matrices having centro-symmetric property $[8,11]$.

Proof : Let $\mathbf{E}_{1}(z)$ be expressed as $\mathbf{E}_{1}(z)=\mathrm{e}(0)+$ $\mathrm{e}(1) z^{-1}$, where $\mathrm{e}(0)$ and $\mathrm{e}(1)$ be partitioned as in (3)

$$
\left(\begin{array}{cc}
\mathrm{e}_{s, l}(0) & \mathrm{e}_{s, r}(0) \\
\mathrm{e}_{a, l}(0) & \mathrm{e}_{a, r}(0) \\
\mathrm{e}_{d, l}(0) & 0
\end{array}\right),\left(\begin{array}{ll}
\mathrm{e}_{s, l}(1) & 0 \\
\mathrm{e}_{a, l}(1) & 0 \\
0 & 0
\end{array}\right)
$$

Following a similar procedure of derivation of (12) and employing (5), e(0) and e(1) can be factored as

$$
\left(\begin{array}{ll}
\mathrm{e}_{a, l}^{\prime}(0) & \mathrm{e}_{s, r}^{\prime}(0) \\
\mathrm{e}_{a, l}^{\prime}(0) & \mathrm{e}_{a, r}^{\prime}(0) \\
0 & \mathrm{e}_{s, r}^{\prime \prime}(0) \\
0 & \mathrm{e}_{a, r}^{\prime \prime}(0) \\
\mathrm{e}_{d, l}(0) & 0
\end{array}\right),\left(\begin{array}{ll}
\mathrm{e}_{s, l}^{\prime}(1) & 0 \\
\mathrm{e}_{a, l}^{\prime}(1) & 0 \\
0 & 0 \\
0 & 0 \\
0 & 0
\end{array}\right)
$$

where $\operatorname{rank}\left(\mathbf{e}_{s, l}^{\prime}(0)\right)=\varsigma^{\prime}$. Proposition 2.1, 2.2 and 2.3 show that the numbers of symmetric and anti-symmetric filters in $\mathbf{E}_{s}^{\prime}(z)$ and $\mathbf{E}_{a}^{\prime}(z)$ are equal; $\mathbf{E}_{s}^{\prime}(z)$ and $\mathbf{E}_{a}^{\prime}(z)$ consist of balanced number of symmetric and anti-symmetric filters, i.e., the number of symmetric filters is equal to or greater than the number of anti-symmetric filters by $1 ; \mathbf{E}_{d}(z)$ itself consists of balanced number of symmetric and antisymmetric filters. Pairwisely combining these symmetric and anti-symmetric rows to pairwise timereversed rows, $e(0)$ and $e(1)$ become

$$
\left(\begin{array}{lr}
\mathrm{e}_{c, l}^{\prime}(0) & \mathrm{e}_{c, r}^{\prime}(0) \\
0 & \mathrm{e}_{c, r}^{\prime \prime}(0) \\
\mathbf{e}_{d, l}^{\prime}(0) & \mathbf{0}
\end{array}\right),\left(\begin{array}{ll}
\mathbf{e}_{c, l}^{\prime}(1) & \mathbf{0} \\
\mathbf{0} & 0 \\
0 & 0
\end{array}\right)
$$

We focus on the left hand side $\beta$ elements in $\mathrm{e}_{c, l}^{\prime}(0)$, $\mathrm{e}_{d, l}^{\prime}(0)$ and $\mathrm{e}_{c, l}^{\prime}(1)$. The centro-symmetric rows in $\mathrm{e}_{d, I}^{\prime}(0)$ form a basis for a $\mu_{0}+\nu_{0}$ dimensional space. With a centro-symmetric matrix performing linear combinations of columns of $\mathbf{e}_{d, l}^{\prime}(0)$, we can turn the central $\mu_{0}+\nu_{0}$ columns of $e_{d, l}^{\prime}(0)$ into a full-ranked centro-symmetric matrix $e_{d}^{\prime \prime}(0)$ while at the same time automatically set the polyphase components to the left, right and above it to zeros. In particular, e(0) and e(1) become

$$
\left(\begin{array}{lccc}
\mathrm{e}_{c, 1}^{\prime \prime \prime}(0) & 0 & \mathrm{e}_{c, 2}^{\prime \prime \prime}(0) & \mathrm{e}_{c, r}^{\prime}(0) \\
0 & 0 & 0 & \mathrm{e}_{c, r}^{\prime \prime}(0) \\
0 & \mathbf{e}_{d}^{\prime \prime}(0) & 0 & 0
\end{array}\right)
$$




$$
\left(\begin{array}{llcl}
\mathrm{e}_{c, 3}^{\prime \prime \prime}(1) & 0 & \mathrm{e}_{c, 4}^{\prime \prime \prime}(1) & \mathbf{0} \\
\mathbf{0} & 0 & \mathbf{0} & \mathbf{0} \\
\mathbf{0} & \mathbf{0} & \mathbf{0} & \mathbf{0}
\end{array}\right) .
$$

The columns of $\mathbf{e}_{c, 1}^{\prime \prime \prime}(0), \mathbf{e}_{c, 2}^{\prime \prime \prime}(0), \mathbf{e}_{c, 3}^{\prime \prime \prime}(1)$ and $\mathbf{e}_{c, 4}^{\prime}(1)$ satisfy the pairwise time-reversed property. Similar to the order reduction process in (14), we can cascade $\mathbf{A}_{1}(z)=\mathbf{W}_{1} \boldsymbol{\Lambda}(z)$ to the right of $\mathbf{e}(0)$ and $\mathbf{e}(1)$, turning $\mathbf{e}(0)$ into

$$
\mathrm{e}(0)=\left(\begin{array}{lccr}
\mathbf{e}_{c, 1}^{\prime \prime \prime}(0) & \mathbf{0} & \mathrm{e}_{c, 2}^{\prime \prime \prime}(0) & \mathrm{e}_{c, r}^{\prime}(0) \\
\mathbf{0} & \mathbf{0} & \mathbf{0} & \mathrm{e}_{s, r}^{\prime \prime}(0) \\
\mathbf{0} & \mathbf{e}_{d}^{\prime \prime}(0) & \mathbf{0} & \mathbf{0}
\end{array}\right)
$$

and $\mathbf{e}(1)$ into $\mathbf{0}$. The difference between $\mathbf{A}_{1}(z)=$ $\mathbf{W}_{1} \boldsymbol{\Lambda}(z)$ and $\mathbf{A}(z)$ in (14) lies in that $\boldsymbol{\Lambda}(z)$ is placed to the right of $\mathbf{W}_{1}$. Furthermore, permuting $\mathbf{e}_{c, r}^{\prime}(0)$ and $\mathbf{e}_{s, r}^{\prime}(0)$ gives

$$
\mathbf{e}(0)=\left(\begin{array}{lccr}
\mathbf{e}_{c, 1}^{\prime \prime \prime}(0) & \mathbf{e}_{c, r}^{\prime}(0) & \mathbf{e}_{c, 2}^{\prime \prime \prime}(0) & \mathbf{0} \\
\mathbf{0} & \mathbf{e}_{3, r}^{\prime}(0) & 0 & \mathbf{0} \\
\mathbf{0} & \mathbf{0} & \mathbf{0} & \mathbf{e}_{d}^{\prime \prime}(0)
\end{array}\right)
$$

Note that the row in $e_{s, r}^{\prime}(0)$ are orthonormal. Similar to the deduction of $(21)$, with a centro-symmetric matrix performing linear combinations of columns of $\mathrm{e}_{s, r}^{\prime}(0)$, we can turn the central $M-\left(\mu_{0}+\nu_{0}\right)-$ $2 \varsigma^{\prime}$ columns of $\mathbf{e}_{s, r}^{\prime}(0)$ into a full-ranked centrosymmetric matrix $\mathbf{e}_{s, r}^{\prime \prime}(0)$ while at the same time automatically set the polyphase components to the left, right and above it to zeros, i.e.,

$$
\mathbf{e}(0)=\left(\begin{array}{lccr}
\mathbf{e}_{c, 1}^{\prime \prime \prime \prime}(0) & 0 & \mathrm{e}_{c, 2}^{\prime \prime \prime \prime}(0) & 0 \\
\mathbf{0} & \mathbf{e}_{s, r}^{\prime \prime}(0) & \mathbf{0} & 0 \\
\mathbf{0} & 0 & \mathbf{0} & \mathrm{e}_{d}^{\prime \prime}(0)
\end{array}\right) .
$$

With column-wise permutations, $\mathbf{e}_{c, 1}^{\prime \prime \prime \prime}(0)$ and $\mathrm{e}_{c, 2}^{\prime \prime \prime \prime}(0)$ can be merged into a centro-symmetric matrix $\mathbf{e}_{c}$. $\mathrm{e}(0)$ becomes

$$
\boldsymbol{\Omega}_{0}=\mathrm{e}(0)=\left(\begin{array}{lll}
\mathrm{e}_{c}(0) & & \\
& \mathrm{e}_{s, r}^{\prime \prime}(0) & \\
& & \mathrm{e}_{d d}^{\prime \prime}(0)
\end{array}\right)
$$

where $\mathrm{e}_{c}(0), \mathrm{e}_{s, r}^{\prime \prime}(0)$ and $\mathrm{e}_{d}^{\prime \prime}(0)$ are centro-symmetric.

\section{Conclusion and design example}

We have presented a new algorithm for designing a family of general linear-phase paraunitary. The lattice factorization is obtained through a succession of "order reduction" processes, compromising planar rotations which force more than half of the rows to zeros, and delay chains which shift the shorten filters to lower orders. A 9-channel filter bank with length $\{32,32,32,32,32,32,23,23,23\}$ and alternative symmetric and anti-symmetric property is shown in Fig 2.

\section{References}

[1] J. D. Johnston, "A filter family designed for use in quadrature mirror filter banks," in Proc. IEEE Int. Conf. on ASSP, Apr. 1980, pp. 291-294.

[2] J. Woods and S. O'Neil, "Subband coding of images," IEEE Trans. on ASSP, pp. 1278-1288, 1986.

[3] S. Mallat, "A theory for multiresolution signal decomposition: The wavelet representation," IEEE Trans. on PAMI, pp. 674-693, 1989.

[4] T. Q. Nguyen and P. P. Vaidyanathan, "Two-channel perfect-reconstruction FIR QMF structures which yield linear-phase analysis and synthesis filters," IEEE Trans. on $A S S P$, pp. $676-690,1989$.

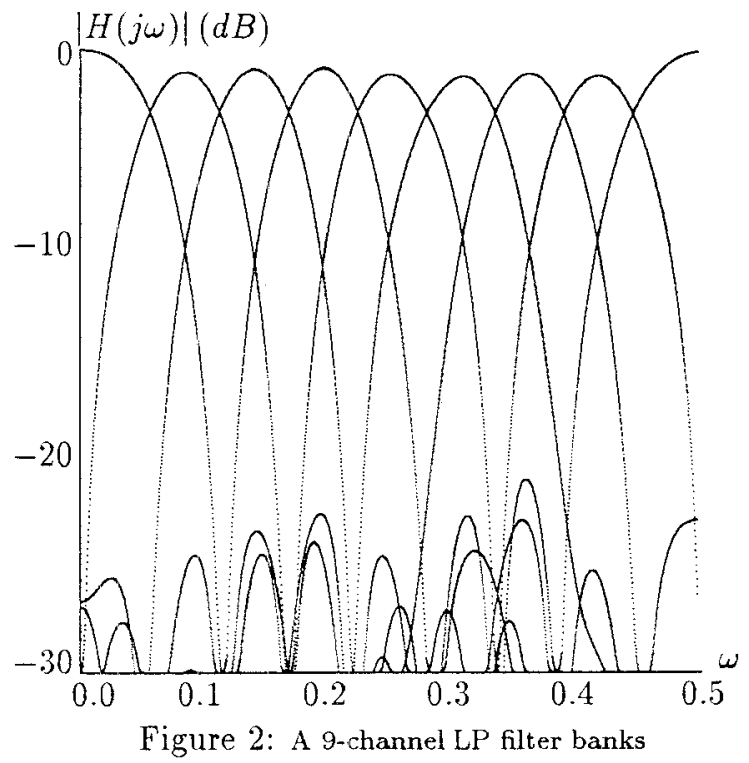

[5] M. Vetterli, "Filter banks allowing perfect reconstruction," Signal Processizg, pp. 219-244, Apr. 1986.

[6] T. Q. Nguyen and P. P. Vaidyanathan, "Structures for M-channel perfect-reconstruction FIR QMF banks which yield linear-phase analysis filters," IEEE Trans. ASSP, pp. 433-446, Mar. 1990.

[7] S. Basu and H. M. Choi, "Hermite-like reduction method for linear phase perfect reconstruction filter bank design," in Proc. IEEE Int. Conf. on ASSP, 1995, pp. 1521-1515.

[8] A. K. Soman, P. P. Vaidyanathan, and T. Q. Nguyen, "Linear phase paraunitary filter banks: Theory, factorizations and applications," IEEE Trans. on Signal Processing, pp. 3480-3496, 1993.

[9] R. L. de Queiroz, T. Q. Nguyen, and K. R. Rao, "The GenLOT: Generalized linear phase lapped orthogonal transform," in IEEE Trans. on Signal Processing, March, 1996.

[10] M. Vetterli and D. Le Gall, "Perfect reconstruction FIR filter banks: Some properties and factorizations," IEEE Trans. on ASSP, pp. 1057-1071, 1989.

[11] R. Turcajová, "Linear phase paraunitary filter banks: A complete characterization," Technical reports Flinders University.

[12] R. Turcajová and J. Kautsky, "Shift products and factorizations of wavelet matrices," Numerical Algorithms, vol. 8, pp. $27-54,1995$.

[13] L. Chen, T. Q. Nguyen, and K. P. Chan, "Linear phase paraunitary filter banks with pairwise mirror image frequency responses," in Proc. of the Twenty-ninth Conference on Information Science and Systems, Maryland, 1995 , pp. 573-578.

[14] T. D. Tran and T. Q. Nguyen, "On $M$-channel linear phase FIR filter banks and application in image compression," submitted to IEEE Trans. Signal Processing, 1996.

[15] H. S. Malvar, Signal Processing with Lapped Transforms. Norwood, MA: Artech House, 1992.

[16] J. Princen and A. Bradley, "Analysis/synthesis filter bank design based on time-domain aliasing cancellation," IEEE Trans. on ASSP, pp. 1153-1161, 1986.

[17] L. Chen, T. Q. Nguyen, and K. P. Chan, "Symmetric extension methods for M-channel linear phase perfect reconstruction filter barks," IEEE Trans. Signal Processing, pp. 2505-2511, 1995.

[18] R. H. Bamberger, S. L. Eddins, and V. Nuri, "Generalized symmetric extension for size-limited multirate filter banks," IEEE Trans. Image Processing, pp. 82-86, 1994. 